\title{
A Kiefer-Wolfowitz Algorithm with Randomized Differences
}

\author{
H. F. Chen, Fellow, IEEE, T. E. Duncan, Fellow, IEEE, and B. Pasik-Duncan, Senior Member, IEEE
}

\begin{abstract}
A Kiefer-Wolfowitz or simultaneous perturbation algorithm that uses either one-sided or two-sided randomized differences and truncations at randomly varying bounds is given in this paper. At each iteration of the algorithm only two observations are required in contrast to $2 \ell$ observations, where $\ell$ is the dimension, in the classical algorithm. The algorithm given here is shown to be convergent under only some mild conditions. A rate of convergence and an asymptotic normality of the algorithm are also established.
\end{abstract}

Index Terms-Kiefer-Wolfowitz algorithm, perturbation algorithm, simultaneous stochastic approximation, stochastic approximation with randomized differences.

\section{INTRODUCTION}

A Kiefer-Wolfowitz (KW) algorithm [8] is used to find the extrema of an unknown function $L: \mathbb{R}^{p} \rightarrow \mathbb{R}$ which may be observed with some additive noise. If the gradient of $L$ can be observed, then the problem can be solved by a Robbins-Monro (RM) algorithm.

Let $x_{n}$ be the estimate of the unique extremum of $L$ at the $n$th iteration. One approach to a $\mathrm{KW}$ algorithm is to observe $L$ at the following values:

$$
\begin{aligned}
& x_{n}^{i+}=\left[x_{n}^{1}, \cdots, x_{n}^{i-1}, x_{n}^{i}+c_{n}, x_{n}^{i+1}, \cdots, x_{n}^{\ell}\right]^{T} \\
& x_{n}^{i-}=\left[x_{n}^{1}, \cdots, x_{n}^{i-1}, x_{n}^{i}-c_{n}, x_{n}^{i+1}, \cdots, x_{n}^{\ell}\right]^{T}
\end{aligned}
$$

for $i=1,2, \cdots, \ell$ where $c_{n} \in \mathbb{R} \backslash\{0\}$.

Consider noisy observations of $L$ so that the processes $\left(y_{n+1}^{i+}, n \in \mathbb{N}, i \in\{1, \cdots, \ell\}\right)$ and $\left(y_{n+1}^{i-}, n \in \mathbb{N}, i \in\right.$ $\{1, \cdots, \ell\})$ satisfy

$$
y_{n+1}^{i+}=L\left(x_{n}^{i+}\right)+\xi_{n+1}^{i+}
$$

and

$$
y_{n+1}^{i-}=L\left(x_{n}^{i-}\right)+\xi_{n+1}^{i-}
$$

where $\left(\xi_{n+1}^{i+}, n \in \mathbb{N}\right)$ and $\left(\xi_{n+1}^{i-}, n \in \mathbb{N}\right)$ are observation noise processes. The ratio

$$
\frac{y_{n+1}^{i+}-y_{n+1}^{i-}}{2 c_{n}}
$$

can be used as an estimate for the $i$ th component of $\nabla L$. Using these estimates a $\mathrm{KW}$ algorithm requires $2 \ell$ measurements of

Manuscript received June 2, 1995; revised August 10, 1997 and May 28, 1998. Recommended by Associate Editor, V. Solo. This work was supported in part by the NSF under Grants DMS 9305936 and DMS 9623439 and the National Natural Science Foundation of China.

H. F. Chen is with the Institute of Systems Science, Beijing 100080, China.

T. E. Duncan and B. Pasik-Duncan are with the Department of Mathematics, University of Kansas, Lawrence, KS 66045 USA (e-mail: duncan@math.ukans.edu).

Publisher Item Identifier S 0018-9286(99)02826-3.
$L$. If $\ell$ is large, for example, the optimization of weights in a neural network, then this $\mathrm{KW}$ algorithm can be rather slow.

To reduce the number of measurements that are required, a random difference method can be used. There is a fairly long history of random search or approximation ideas in stochastic approximation (e.g., Fabian [7]). Koronacki [10] introduced a random version of the $\mathrm{KW}$ algorithm using a sequence of random unit vectors that are independent and uniformly distributed on the unit sphere or unit cube, and he gave sufficient conditions for the convergence of the algorithm. Koronacki [10], [11] noted that the random direction methods have a better reduction of bias effects caused by the use of finite differences for the derivatives than the nonrandom direction methods and that these methods reduce the required number of observations or measurements. Spall [14] reintroduced a random direction version of the $\mathrm{KW}$ algorithm and called it a simultaneous perturbation stochastic approximation (SPSA) algorithm. Using the ordinary differential equation (ODE) method [12] Spall showed the convergence and the asymptotic normality of this modified $\mathrm{KW}$ or simultaneous perturbation algorithm though the conditions that he required are restrictive.

Initially Spall's KW or simultaneous perturbation algorithm and the conditions that he uses are described. Let $\left(\Delta_{k}^{i}\right.$, $i=1, \cdots, \ell, k=1,2, \cdots)$ be a sequence of mutually independent and identically distributed random variables with zero mean. Let $\Delta_{k}$ be given by

$$
\Delta_{k}=\left[\Delta_{k}^{1}, \cdots, \Delta_{k}^{\ell}\right]^{T} .
$$

At each iteration, two measurements are taken:

$$
\begin{aligned}
& y_{k+1}^{+}=L\left(x_{k}+c_{k} \Delta_{k}\right)+\xi_{k+1}^{+} \\
& y_{k+1}^{-}=L\left(x_{k}-c_{k} \Delta_{k}\right)+\xi_{k+1}^{-} .
\end{aligned}
$$

Then the vector symmetric difference

$$
\frac{\left(y_{k+1}^{+}-y_{k+1}^{-}\right) g_{k}}{2 c_{k}}
$$

where

$$
g_{k}=\left[\frac{1}{\Delta_{k}^{1}}, \cdots, \frac{1}{\Delta_{k}^{\ell}}\right]^{T}
$$

is used as an estimate for $\nabla L\left(x_{k}\right)$.

The KW or simultaneous perturbation algorithm is formed as follows:

$$
x_{k+1}=x_{k}+a_{k} \frac{\left(y_{k+1}^{+}-y_{k+1}^{-}\right) g_{k}}{2 c_{k}} .
$$

In this form the algorithm seeks the maximum of $L$. The minimum of $L$ is found by replacing $a_{k}$ by $-a_{k}$. 
For the convergence of the algorithm (5) Spall [14] required the following conditions.

A1) The random variables $\left(\xi_{k+1}^{+}-\xi_{k+1}^{-}, k \in \mathbb{N}\right)$ is a martingale difference sequence (mds) with uniformly bounded second moments.

A2) The integrability condition

$$
\sup _{k \in \mathbb{N}} E\left(L^{2}\left(x_{k}+c_{k} \Delta_{k}\right)\right)<\infty
$$

is satisfied.

A3) The sequence $\left(x_{k}, k \in \mathbb{N}\right)$ is assumed a priori to be uniformly bounded, that is,

$$
\sup _{k \in \mathbb{N}}\left\|x_{k}\right\|<\eta<\infty \quad \text { a.s. }
$$

where $\eta \in \mathbb{R}_{+}$.

A4) The third derivative of $L$ is bounded.

A5) The point $x^{0} \in \mathbb{R}^{\ell}$ is an asymptotically stable point for the differential equation $d x / d t=-f(x(t))$ where $f=\nabla L$.

A6) The sequence $\left(x_{k}, k \in \mathbb{N}\right)$ is infinitely often in a compact set that is contained in the domain of attraction of $x^{0}$ given in A5).

A7) The sequences $\left(a_{k}, k \in \mathbb{N}\right)$ and $\left(c_{k}, k \in \mathbb{N}\right)$ satisfy $a_{k}>0, c_{k}>0$ for all $k \in \mathbb{N}, a_{k} \rightarrow 0$ and $c_{k} \rightarrow 0$ as $k \rightarrow \infty, \sum_{k=1}^{\infty} a_{k}=\infty$ and $\sum_{k=1}^{\infty}\left(a_{k} / c_{k}\right)^{2}<\infty$.

Furthermore, some conditions are imposed on the sequence $\left(\Delta_{k}, k \in \mathbb{N}\right)$ in [14], but this sequence can be arbitrarily chosen by the user of the algorithm so these conditions should not be considered as restrictions.

In this paper, both a one-sided randomized difference and a two-sided randomized difference are used, that is,

$$
\begin{aligned}
& \frac{\left(y_{k+1}^{+}-y_{k+1}^{0}\right) g_{k}}{c_{k}} \\
& \frac{\left(y_{k+1}^{+}-y_{k+1}^{-}\right) g_{k}}{2 c_{k}}
\end{aligned}
$$

and

$$
g_{k}=\left[\frac{1}{\Delta_{k}^{1}}, \cdots, \frac{1}{\Delta_{k}^{\ell}}\right]^{T}
$$

are used to estimate $f\left(x_{k}\right)=\nabla L\left(x_{k}\right)$ where

$$
y_{k+1}^{0}=L\left(x_{k}\right)+\xi_{k+1}^{0} \text {. }
$$

$y_{k+1}^{+}, y_{k+1}^{-}$, and $g_{k}$ are given by (3), (4), and (6), respectively.

The two-sided algorithm is described by (7) and the onesided algorithm is described by (7) with $\left(8^{\prime}\right)$ replaced by (8). By a modification of the algorithm (7) and the use of a direct approach to verifying convergence, the conditions A2), A3), A5), and A6) are eliminated, A4) and A7) are relaxed, and A1) is weakened to one that provides not only a sufficient but also a necessary condition for convergence. This result is given in Theorem 1. In Theorem 2 the observation noise is modified to one with only bounded second moments that is independent of $\left(\Delta_{k}, k \in \mathbb{N}\right)$. A convergence rate and an asymptotic normality of the algorithm are given in Theorems 3 and 4, respectively.

\section{The Algorithm AND ITS CONVERGENCE}

Initially the algorithm is precisely described. Let $\left(\Delta_{k}^{i}, i=\right.$ $1, \cdots, \ell, k \in \mathbb{N}$ ) be a sequence of independent and identically distributed random variables where $\left|\Delta_{k}^{i}\right|<a,\left|1 / \Delta_{k}^{i}\right|<b$, $E\left(1 / \Delta_{k}^{i}\right)=0$ for all $i \in\{1, \cdots, \ell\}$ and $k \in \mathbb{N}$ and some $a$, $b \in \mathbb{R}_{+}$. Furthermore, let $\Delta_{k}$ be independent of $\mathcal{F}_{k}^{\xi}=\sigma\left(\xi_{i}^{+}\right.$, $\left.\xi_{i}^{0}, \xi_{i}^{-}, i \in\{0, \cdots, k\}\right)$ for $k \in \mathbb{N}$. Define $y_{k+1}$ and $\xi_{k+1}$ by the following equations:

$$
\begin{aligned}
y_{k+1} & =\frac{\left(y_{k+1}^{+}-y_{k+1}^{0}\right) g_{k}}{c_{k}} \\
y_{k+1} & =\frac{\left(y_{k+1}^{+}-y_{k+1}^{-}\right) g_{k}}{2 c_{k}} \\
\xi_{k+1} & =\xi_{k+1}^{+}-\xi_{k+1}^{0} \\
\xi_{k+1} & =\xi_{k+1}^{+}-\xi_{k+1}^{-} .
\end{aligned}
$$

It follows that

$$
\begin{aligned}
y_{k+1} & =\frac{\left(L\left(x_{k}+c_{k} \Delta_{k}\right)-L\left(x_{k}\right)\right) g_{k}}{c_{k}}+\frac{\xi_{k+1} g_{k}}{c_{k}} \\
y_{k+1} & =\frac{\left(L\left(x_{k}+c_{k} \Delta_{k}\right)-L\left(x_{k}-c_{k} \Delta_{k}\right)\right) g_{k}}{2 c_{k}}+\frac{\xi_{k+1} g_{k}}{2 c_{k}}
\end{aligned}
$$

Equations (10), $\left(10^{\prime}\right),(11),\left(11^{\prime}\right),(12)$, and $\left(12^{\prime}\right)$ represent some abuse of notation. Equations (10)-(12) are used for the one-sided algorithm and $\left(10^{\prime}\right),\left(11^{\prime}\right)$, and $\left(12^{\prime}\right)$ are used for the two-sided algorithm. In the subsequent description the meaning of $y_{k+1}$ is determined by whether the one-sided or the two-sided algorithm is used. Choose $x^{*} \in \mathbb{R}^{\ell}$ and fix it. Define the following $\mathrm{KW}$ algorithm with randomly varying truncations and randomized differences:

$$
\begin{aligned}
x_{k+1}= & \left(x_{k}+a_{k} y_{k+1}\right) 1_{\left\{\left\|x_{k}+a_{k} y_{k+1}\right\| \leq M_{\sigma_{k}}\right\}} \\
& +x^{*} 1_{\left\{\left\|x_{k}+a_{k}, y_{k+1}\right\|>M_{\sigma_{k}}\right\}} \\
\sigma_{k}= & \sum_{i=0}^{k-1} 1_{\left\{\left\|x_{i}+a_{i} y_{i+1}\right\|>M_{\sigma_{i}}\right\}} \\
\sigma_{0} \equiv & 0
\end{aligned}
$$

where $\left(M_{k}, k \in \mathbb{N}\right)$ is a sequence of strictly positive, strictly increasing real numbers that diverges to $+\infty$. It is clear that $\sigma_{k}$ is the number of truncations that have occurred before time $k$. Clearly the random vector $x_{k}$ is measurable with respect to $\mathcal{F}_{k}:=\mathcal{F}_{k}^{\xi} \vee \mathcal{F}_{k-1}^{\Delta}$ where $\mathcal{F}_{k}^{\Delta}=\sigma\left(\Delta_{i}, i \in\{0, \cdots, k\}\right)$. Thus the random vector $\Delta_{k}$ is independent of $\sigma\left(x_{i}, i \leq k\right)$.

The following conditions are imposed on the algorithm.

H1) The function $\nabla L=f$ is locally Lipschitz continuous. There is a unique maximum of $L$ at $x^{0}$ that is the only local extremum so that $f\left(x^{0}\right)=0$ and $f(x) \neq 0$ for $x \neq x^{0}$. There is a $c_{0} \in \mathbb{R}_{+}$such that $\left\|x^{*}\right\|<c_{0}$ and $\sup _{\|x\|=c_{0}} L(x)<L\left(x^{*}\right)$.

H2) The two sequences of strictly positive real numbers $\left(a_{k}, k \in \mathbb{N}\right)$ and $\left(c_{k}, k \in \mathbb{N}\right)$ satisfy $a_{k} \rightarrow 0$ and $c_{k} \rightarrow 0$ as $k \rightarrow \infty, \sum_{k=1}^{\infty} a_{k}=\infty$ and there is a $p \in(1,2]$ such that $\sum_{k=1}^{\infty} a_{k}^{p}<\infty$.

Remark: If $L$ is twice continuously differentiable then $f$ is locally Lipschitz continuous. If in H1) $x^{0}$ is the unique minimum of $L$, then in (13) and (14) $a_{k}$ should be replaced by $-a_{k}$. 
The main result of this section is the following theorem that gives necessary and sufficient conditions for the convergence of the algorithm (13).

Theorem 1: Let $\mathrm{H} 1)$ and $\mathrm{H} 2)$ be satisfied and $\left(x_{k}, k \in \mathbb{N}\right)$ be given by (13) with the conditions for $\left(\Delta_{k}^{i}, i=1, \cdots, \ell\right.$; $k \in \mathbb{N})$ given for the algorithm. The sequence $\left(x_{k}, k \in \mathbb{N}\right)$ satisfies

$$
\lim _{k \rightarrow \infty} x_{k}=x^{0} \quad \text { a.s. }
$$

where $x^{0}$ is given in $\left.\mathrm{H} 1\right)$ if and only if for each $k \in \mathbb{N}$ the observation noise $\xi_{k}$ in (11) or $\left(11^{\prime}\right)$ can be decomposed into the sum of two terms for each $j \in\{1, \cdots, \ell\}$ as

$$
\xi_{k}=e_{k}^{j}+\nu_{k}^{j}
$$

such that

$$
\sum_{k=1}^{\infty} \frac{a_{k} e_{k+1}^{j}}{c_{k} \Delta_{k}^{j}}<\infty \quad \text { a.s. }
$$

and

$$
\lim _{k \rightarrow \infty} \frac{\nu_{k+1}^{j}}{c_{k} \Delta_{k}^{j}}=0 \quad \text { a.s. }
$$

for $j=1, \cdots, \ell$ where $\Delta_{k}^{j}$ is given in (2).

Before proving the theorem, two lemmas are proved.

To describe easily the replacement of some components of $\Delta_{k}$ by zero, two functions are introduced. Let $\Delta_{k}(\cdot)$ $\{0, \cdots, \ell\} \rightarrow \mathbb{R}^{l}$ and $\Delta_{k}^{c}(\cdot)\{0, \cdots, \ell\} \rightarrow \mathbb{R}^{\ell}$ be given by

$$
\begin{aligned}
& \Delta_{k}(s)=\left[\Delta_{k}^{1}, \cdots, \Delta_{k}^{s}, 0, \cdots, 0\right]^{T}, \quad \Delta_{k}(0)=0 \\
& \Delta_{k}^{c}(s)=\left[0, \cdots, 0, \Delta_{k}^{s+1}, \cdots, \Delta_{k}^{\ell}\right]^{T}, \quad \Delta_{k}^{c}(\ell)=0 .
\end{aligned}
$$

It is clear that

$$
\Delta_{k}=\Delta_{k}^{c}(0)=\Delta_{k}(\ell)
$$

and

$$
\Delta_{k}(i-1)+\Delta_{k}^{c}(i)=\left[\Delta_{k}^{1}, \cdots, \Delta_{k}^{i-1}, 0, \Delta_{k}^{i+1}, \cdots, \Delta_{k}^{\ell}\right]^{T} .
$$

For notational convenience let $\delta_{k}(i)$ denote a generic $\mathbb{R}^{\ell}$ valued random vector such that

$$
\delta_{k}(i)=[\underbrace{0, \cdots, 0}_{i-1}, \delta_{k}^{i}, \underbrace{0, \cdots, 0}_{\ell-i}]^{T}
$$

where $\left|\delta_{k}(i)\right| \leq\left|c_{k} a\right|, a \in \mathbb{R}^{\ell}$ is fixed, and $\delta_{k}^{i}$ may vary for different applications.

Lemma 1: If H1) is satisfied, then the observation $y_{k+1}$ given by (12) or $\left(12^{\prime}\right)$ can be expressed as

$$
y_{k+1}=f\left(x_{k}\right)+\varepsilon_{k+1}
$$

where

$$
\varepsilon_{k+1}=w_{k}(1)+w_{k}(2)+\frac{1}{c_{k}} \xi_{k+1} g_{k}
$$

for $y_{k+1}$ given by (12) and

$$
\varepsilon_{k+1}=\frac{1}{2}\left(w_{k}(1)+w_{k}(2)+u_{k}(1)+u_{k}(2) \frac{1}{c_{k}} \xi_{k+1} g_{k}\right)
$$

for $y_{k+1}$ given by $\left(12^{\prime}\right)$ and the $i$ th components of $w_{k}(1)$ and $u_{k}(1)$ are, respectively

$$
\begin{gathered}
w_{k, i}^{i}=f_{i}\left(x_{k}+c_{k} \Delta_{k}+\delta_{k}(i)\right)-f_{i}\left(x_{k}\right) \\
u_{k, i}^{i}=f_{i}\left(x_{k}+c_{k} \Delta_{k}+\delta_{i}(i)\right)-f_{i}\left(x_{k}\right) \\
f_{i}\left(x_{k}\right)=\frac{\partial L\left(x_{k}\right)}{\partial x_{k}^{i}}
\end{gathered}
$$

and the $i$ th components of $w_{k}(2)$ and $u_{k}(2)$ are $\sum_{j \neq i}^{\ell} h_{k j}\left(1 / \Delta_{k}^{i}\right)$ and $\sum_{j \neq i}^{\ell} g_{k j}\left(1 / \Delta_{k}^{i}\right)$, respectively, and

$$
\begin{aligned}
& h_{k j}=\left\{\begin{array}{c}
f_{j}\left(x_{k}+c_{k} \Delta_{k}(j-1)+\delta_{k}(j)\right) \Delta_{k}^{j} \\
j \in\{1, \cdots, i-1\} \\
f_{j}\left(x_{k}+c_{k}\left(\Delta_{k}(i-1)+\Delta_{k}^{c}(j)\right)+\delta_{k}(j)\right) \Delta_{k}^{j} \\
j \in\{i+1, \cdots, \ell\}
\end{array}\right. \\
& g_{k j}=\left\{\begin{array}{c}
f_{j}\left(x_{k}-c_{k} \Delta_{k}(j-1)+\delta_{k}(j)\right) \Delta_{k}^{j} \\
j \in\{1, \cdots, i-1\} \\
f_{j}\left(x_{k}-c_{k}\left(\Delta_{k}(i-1)+\Delta_{k}^{c}(j)\right)+\delta_{k}(j)\right) \Delta_{k}^{j} \\
j \in\{i+1, \cdots, \ell\}
\end{array}\right.
\end{aligned}
$$

and $h_{k j}$ and $g_{k j}$ are independent of $\Delta_{k}^{i}$.

Proof: By comparing $\left(12^{\prime}\right)$ and (24) it follows that the $i$ th component of $\epsilon_{k+1}$ is

$$
\frac{L\left(x_{k}+c_{k} \Delta_{k}\right)-L\left(x_{k}-c_{k} \Delta_{k}\right)}{2 c_{k} \Delta_{k}^{i}}-f_{i}\left(x_{k}\right)+\frac{1}{2 c_{k} \Delta_{k}^{i}} \xi_{k+1} .
$$

Therefore, to obtain (26) it suffices to show that

$$
\begin{gathered}
\frac{L\left(x_{k}+c_{k} \Delta_{k}\right)-L\left(x_{k}-c_{k} \Delta_{k}\right)}{2 c_{k} \Delta_{k}^{i}}-f_{i}\left(x_{k}\right) \\
=\frac{1}{2}\left[w_{k i}^{i}+u_{k i}^{i}+\sum_{j \neq i}^{l}\left(w_{k i}^{j}+u_{k i}^{j}\right)\right]
\end{gathered}
$$

where

$$
w_{k i}^{j}=h_{k j} \frac{1}{\Delta_{k}^{i}}
$$

and

$$
u_{k i}^{j}=g_{k j} \frac{1}{\Delta_{k}^{i}} .
$$

It follows that

$$
\begin{aligned}
& \frac{L\left(x_{k}+c_{k} \Delta_{k}\right)-L\left(x_{k}-c_{k} \Delta_{k}\right)}{2 c_{k} \Delta_{k}^{i}}-f_{i}\left(x_{k}\right) \\
& =\frac{L\left(x_{k}+c_{k} \Delta_{k}\right)-L\left(x_{k}\right)}{2 c_{k} \Delta_{k}^{i}}+\frac{L\left(x_{k}\right)-L\left(x_{k}-c_{k} \Delta_{k}\right)}{2 c_{k} \Delta_{k}^{i}} \\
& -f_{i}\left(x_{k}\right) \\
& =\frac{1}{2 c_{k} \Delta_{k}^{i}}\left\{\begin{array}{l}
\sum_{j=1}^{i-1}\left[L\left(x_{k}+c_{k} \Delta_{k}(j)\right)\right. \\
\left.-L\left(x_{k}+c_{k} \Delta_{k}(j-1)\right)\right]+L\left(x_{k}+c_{k} \Delta_{k}\right) \\
-L\left(x_{k}+c_{k}\left(\Delta_{k}(i-1)+\Delta_{k}^{c}(i)\right)\right) \\
\quad+\sum_{j=i+1}^{l}\left[L\left(x_{k}+c_{k}\left(\Delta_{k}(i-1)+\Delta_{k}^{c}(j-1)\right)\right)\right. \\
-L\left(x_{k}+c_{k}\left(\Delta_{k}(i-1)+\Delta_{k}^{c}(j)\right)\right] \\
\left.-L\left(x_{k}+c_{k}\left(\Delta_{k}(i-1)+\Delta_{k}^{c}(j)\right)\right)\right]
\end{array}\right\}
\end{aligned}
$$




$$
\begin{aligned}
+\frac{1}{2 c_{k} \Delta_{k}^{i}} & \left\{\sum _ { j = 1 } ^ { i - 1 } \left[L\left(x_{k}-c_{k} \Delta_{k}(j-1)\right)\right.\right. \\
& \left.-L\left(x_{k}-c_{k} \Delta_{k}(j)\right)\right] \\
& +\sum_{j=i+1}^{l}\left[L\left(x_{k}-c_{k}\left(\Delta_{k}(i-1)+\Delta_{k}^{c}(j)\right)\right)\right. \\
& \left.-L\left(x_{k}-c_{k}\left(\Delta_{k}(i-1)+\Delta_{k}^{c}(j-1)\right)\right)\right] \\
& +L\left(x_{k}-c_{k}\left(\Delta_{k}(i-1)+\Delta_{k}^{c}(c)\right)\right) \\
& \left.-L\left(x_{k}-c_{k} \Delta_{k}\right)\right\} \\
-f_{i}\left(x_{k}\right) &
\end{aligned}
$$

It is clear by the Taylor formula that

$$
\begin{aligned}
& \frac{1}{2}\left[\frac{L\left(x_{k}+c_{k} \Delta_{k}\right)-L\left(x_{k}+c_{k}\left(\Delta_{k}(i-1)+\Delta_{k}^{c}(i)\right)\right)}{c_{k} \Delta_{k}}\right. \\
& \quad+\frac{L\left(x_{k}-c_{k}\left(\Delta_{k}(i-1)+\Delta_{k}^{c}(i)\right)\right)-L\left(x_{k}-c_{k} \Delta_{k}\right)}{c_{k} \Delta_{k}^{i}} \\
& \left.\quad-2 f_{i}\left(x_{k}\right)\right]=w_{k i}^{i}+u_{k i}^{i}
\end{aligned}
$$

and for $j \leq i-1, L\left(x_{k}+c_{k} \Delta(j)\right)-L\left(x_{k}+c_{k} \Delta_{k}(j-1)\right)=$ $f_{j}\left(x_{k}+c_{k} \Delta(j-1)+\Delta_{k}(j)\right) \Delta_{k}^{j}=h_{k j}$, which is independent of $\Delta_{k}^{i}$.

The other expressions in (29) and (30) are obtained by a similar argument.

For $y_{k+1}$ given by (12), (31) changes to

$$
\frac{L\left(x_{k}+c_{k} \Delta_{k}\right)-L\left(x_{k}\right)}{c_{k} \Delta_{k}^{i}}-f_{i}\left(x_{k}\right)=w_{k i}^{i}+\sum_{j \neq i}^{l} w_{k i}^{j}
$$

and the analysis is the same except that the $u_{k i}^{j}$ terms are deleted.

Lemma 2: If H1), H2), (17), and (18) are satisfied, then there is $\Omega^{\prime} \subset \Omega$ with $P\left(\Omega^{\prime}\right)=1$ such that for any fixed $\omega \in \Omega^{\prime}$ and any convergent subsequence $\left\{x_{n_{k}}(\omega), k \in \mathbb{N}\right\}$ there exist positive constants $M, T$, and $k_{T}$ such that if $k>k_{T}$, then

$$
\left\|\sum_{i=n_{k}}^{m+1} a_{i} y_{i+1}(\omega)\right\| \leq M
$$

for each $m \in\left\{n_{k}, \cdots, m\left(n_{k}, T\right)\right\}$, where

$$
m(n, t)=\max \left\{m: \sum_{i=n}^{m} a_{i} \leq t\right\} .
$$

Proof: For notational convenience, the evaluation of the random variables at $\omega$ is suppressed. For example, $x_{n_{k}}(\omega)$ is simply denoted as $x_{n_{k}}$.

Since $h_{k j}$ and $g_{k j}$ are independent of $\Delta_{k}^{i}$ and $\sum_{i=1}^{\infty} a_{i}^{p}<\infty$ for some $p \in(1,2]$ it follows by the convergence theorem for mds's [3], [6] that for each $N \in \mathbb{N}$ and $j \neq i$

$$
\begin{aligned}
& \sum_{k=1}^{\infty} a_{k} w_{k i}^{j} 1_{\left\{\left|h_{k j}\right| \leq N\right\}}<\infty, \quad \text { a.s. } \\
& \sum_{k=1}^{\infty} a_{k} u_{k i}^{j} 1_{\left\{\left|g_{k j}\right| \leq N\right\}}<\infty, \quad \text { a.s. }
\end{aligned}
$$

Let $\Omega^{\prime} \subset \Omega$ be the $\omega$-set such that for each $\omega \in \Omega^{\prime}$ (17) is finite, (18) is satisfied, and (38) and (39) are finite for all $N \in \mathbb{N}$. It is clear that $P\left(\Omega^{\prime}\right)=1$.

If the number of truncations is finite then there is an $N$ such that $\sigma_{n}=\sigma_{N}$ for all $n \geq N$ and $\left\|\sum_{i=n_{k}}^{m+1} a_{i} y_{i+1}\right\|=$ $\left\|x_{m+2}-x_{n_{k}}\right\| \leq 2 M_{\sigma_{N}}$ for all $n_{k} \geq N$. Thus the inequality (36) is satisfied.

Now let $\sigma_{n} \rightarrow \infty$ and assume that (36) is not satisfied. Choose $c>\|\bar{x}\|$ and fix it where $\bar{x}$ is the limit of the subsequence, $\left(x_{n_{k}}, k \in \mathbb{N}\right)$. By the convergence of $\left(x_{n_{k}}\right.$, $k \in \mathbb{N}$ ) there is a $k_{c}$ such that

$$
\left\|x_{n_{k}}\right\|<\frac{c+\|\bar{x}\|}{2}
$$

for all $k \geq k_{c}$. Choose a sequence $\left(T_{s}, s \in \mathbb{N}\right)$ of strictly positive numbers that converges to zero. Since it is assumed that (36) is not satisfied for each $T_{s}$, there are a $k_{s}>s$ and an $m_{s} \in\left\{n_{k_{s}}, \cdots, m\left(n_{k_{s}}, T_{s}\right)\right\}$ such that

$$
\left\|\sum_{i=n_{k_{s}}}^{m_{s}+1} a_{i} y_{i+1}\right\|>\frac{c-\|\bar{x}\|}{2} .
$$

It can be assumed that $k_{s+1}>k_{s} \geq k_{c}$ for all $s \in \mathbb{N}$ and let

$$
m_{s}=\inf \left\{m:\left\|\sum_{i=n_{k_{s}}}^{m+1} a_{i} y_{i+1}\right\|>\frac{c-\|\bar{x}\|}{2}\right\} .
$$

Since the sequence $\left(x_{n_{k}}, k \in \mathbb{N}\right)$ is convergent, it follows by the Lipschitz continuity of $f$ that

$$
\begin{aligned}
& \lim _{k \rightarrow \infty} w_{n_{k}, i}^{i}=0 \\
& \lim _{k \rightarrow \infty} u_{n_{k}, i}^{i}=0
\end{aligned}
$$

for $i \in\{1, \cdots, l\}$ where $w_{k, i}^{i}$ and $u_{k i}^{i}$ satisfy (27) and (28), respectively. It is elementary to verify that

$$
\begin{aligned}
\lim _{k \rightarrow \infty} a_{n_{k}} w_{n_{k}, i}^{j} & =0 \\
\lim _{k \rightarrow \infty} a_{n_{k}} u_{n_{k} i}^{j} & =0
\end{aligned}
$$

for $i \neq j$. Thus

$$
\lim _{s \rightarrow \infty} a_{n_{k_{s}}} y_{n_{k_{s}}+1}=0
$$

where $m_{s} \geq n_{k_{s}}$ and $m_{s}$ is given by (40). From (40) and the convergence of $\left(x_{n_{k}}, k \in \mathbb{N}\right)$ it follows that

$$
\left\|x_{n_{k_{s}}}+\sum_{i=n_{k_{s}}}^{m} a_{i} y_{i+1}\right\| \leq c
$$

for each $m \in\left\{n_{k_{s}}, m_{s}\right\}$.

Since $\sigma_{n} \rightarrow \infty$ as $n \rightarrow \infty$ there is an $s_{0}$ such that if $s \geq s_{0}$ then $M_{\sigma_{n_{k_{s}}}}>c$. Thus

$$
\left\|x_{n_{k_{s}}}+\sum_{i=n_{k_{s}}}^{m} a_{i} y_{i+1}\right\| \leq M_{\sigma_{n_{k_{s}}}}
$$

and

$$
x_{m+1}=x_{m}+a_{m} y_{m+1}
$$


for all $m \in\left\{n_{k_{s}}, \cdots, m_{s}\right\}$. Inequality (41) implies that

$$
\left\|x_{m}\right\| \leq c
$$

and

$$
\left\|f\left(x_{m}\right)\right\| \leq c^{\prime}
$$

for all $m \in\left\{n_{k_{s}}, \cdots, m_{s+1}\right\}$ where $d^{\prime} \in \mathbb{R}_{+}$. By the local Lipschitz condition it follows that

$$
\lim _{s \rightarrow \infty} \max \left\{\left|w_{m j}^{j}\right|+\left|u_{m j}^{j}\right|: m \in\left\{n_{k_{s}}, \cdots, m_{s}+1\right\}\right\}=0
$$

where $w_{k j}^{i}$ and $u_{k j}^{i}$ are given by (32) and (33), respectively, and thus

$$
\begin{aligned}
& \lim _{s \rightarrow \infty} \sum_{i=n_{k_{s}}}^{m_{s}} a_{i} w_{i j}^{j}=0 \\
& \lim _{s \rightarrow \infty} \sum_{i=n_{k_{s}}}^{m_{s}} a_{i} u_{i j}^{j}=0
\end{aligned}
$$

and

$$
\begin{aligned}
& \lim _{s \rightarrow \infty} a_{m_{s}+1} w_{m_{s}+1 j}^{j}=0 \\
& \lim _{s \rightarrow \infty} a_{m_{s}+1} u_{m_{s}+1 j}^{j}=0 .
\end{aligned}
$$

For $N>c^{\prime}$ it follows from (38), (39), (44), and (45) that

and

$$
\begin{aligned}
& \lim _{s \rightarrow \infty} \sum_{i=n_{k_{s}}}^{m_{s}} a_{i} w_{i \lambda}^{j}=0 \\
& \lim _{s \rightarrow \infty} \sum_{i=n_{k_{s}}}^{m_{s}} a_{i} u_{i \lambda}^{j}=0
\end{aligned}
$$

$$
\begin{aligned}
& \lim _{s \rightarrow \infty} a_{m_{s}+1} w_{m_{s}+1 \lambda}^{j}=0 \\
& \lim _{s \rightarrow \infty} a_{m_{s}+1} u_{m_{s}+1 \lambda}^{j}=0
\end{aligned}
$$

for all $\lambda \neq j$ and $j, \lambda \in\{1, \cdots, l\}$. Combining (38), (39), (44), and (45) it follows that

$$
\lim _{s \rightarrow \infty} \sum_{i=n_{k_{s}}}^{m_{s}} a_{i} \epsilon_{i+1}=0
$$

and

$$
\lim _{s \rightarrow \infty} a_{m_{s}+1} \epsilon_{m_{s}+2}=0 .
$$

The following inequalities are elementary:

$$
\begin{aligned}
\left\|x_{m_{s}+1}-x_{n_{k_{s}}}\right\| & \leq \sum_{i=n_{k_{s}}}^{m_{s}} a_{i}\left\|f\left(x_{i}\right)\right\|+\left\|\sum_{i=n_{k_{s}}}^{m_{s}} a_{i} \epsilon_{i+1}\right\| \\
& \leq c^{\prime} T_{s}+\left\|\sum_{i=n_{k_{s}}}^{m_{s}} a_{i} \epsilon_{i+1}\right\|
\end{aligned}
$$

and

$$
\begin{aligned}
& \left\|x_{m_{s}+1}-x_{n_{k_{s}}}+a_{m_{s}+1} y_{m_{s}+2}\right\| \\
& \quad \leq\left\|x_{m_{s}+1}-x_{n_{k_{s}}}\right\|+a_{m_{s}+1}\left\|f\left(x_{m_{s}}+1\right)\right\| \\
& \quad+\left\|a_{m_{s}+1} \epsilon_{m_{s}+2}\right\| .
\end{aligned}
$$

The right-hand sides of the inequalities (46) and (47) tend to zero as $s \rightarrow \infty$. However by (40) it follows that

$\left\|x_{m_{s}+1}-x_{n_{k_{s}}}+a_{m_{s}+1} y_{m_{s}+2}\right\|=\left\|\sum_{i=n_{k_{s}}}^{m_{s}+1} a_{i} y_{i+1}\right\|>\frac{c-\|\bar{x}\|}{2}$

so that (47) and (48) are not compatible and (36) is verified.

Now the proof of Theorem 1 is given.

Proof-Sufficiency: By (36) it follows that for $k$ sufficiently large there is no truncation for all $m \in\left\{n_{k}, \cdots, m\left(n_{k}, T\right)\right\}$ so

$$
x_{m+1}=x_{m}+a_{m} y_{m+1}
$$

and $\left\|x_{m}\right\| \leq 2 M,\left\|f\left(x_{m}\right)\right\| \leq M^{\prime}$ where $M^{\prime} \in \mathbb{R}_{+}$.

By these last two inequalities, there is an $N>0$ such that $h_{m j}$ and $g_{m j}$ given by (29) and (30), respectively, satisfy the inequalities

$$
\begin{aligned}
& \left|h_{m j}\right| \leq N \\
& \left|g_{m j}\right| \leq N
\end{aligned}
$$

for all $m \in\left\{n_{k}, \cdots, m\left(n_{k}, T\right)\right\}$ and $j \in\{1, \cdots, l\}$ for all $k$ sufficiently large. Therefore, for $\lambda \neq j$

$$
\begin{aligned}
& \lim _{k \rightarrow \infty}\left|\sum_{i=n_{k}}^{m\left(n_{k}, t\right)} a_{i} w_{i \lambda}^{j}(\omega)\right| \\
& =\lim _{k \rightarrow \infty}\left|\sum_{i=n_{k}}^{m\left(n_{k}, t\right)} a_{i} w_{i \lambda}^{j}(\omega) 1_{\left\{\left|h_{i j}\right| \leq N\right\}}\right|=0
\end{aligned}
$$

for $\lambda \in\{1, \cdots, l\}$ by (38) and (39) for $\omega \in \Omega^{\prime}$ where $\Omega^{\prime}$ is given in Lemma 2. Thus for $\omega \in \Omega^{\prime}$ and $\lambda \neq j$

$$
\lim _{T \rightarrow 0} \limsup _{k \rightarrow \infty} \frac{1}{T}\left\|\sum_{i=n_{k}}^{m\left(n_{k}, t\right)} a_{i} w_{i \lambda}^{j}\right\|=0
$$

for all $t \in[0, T]$. For $\lambda=j$ it follows that

$$
\begin{aligned}
& \lim _{T \rightarrow 0} \limsup _{k \rightarrow \infty} \frac{1}{T}\left|\sum_{i=n_{k}}^{m\left(n_{k}, t\right)} a_{i} w_{i \lambda}^{\lambda}(\omega)\right| \\
& =\lim _{T \rightarrow 0} \limsup _{k \rightarrow \infty} \frac{1}{T} \mid \sum_{i=n_{k}}^{m\left(n_{k}, t\right)} a_{i}\left(f_{\lambda}\left(x_{i}+c_{i} \Delta_{i}-\delta_{i}(\lambda)\right)\right. \\
& \left.\quad-f_{\lambda}\left(x_{i}\right)\right) \mid \\
& =\lim _{T \rightarrow 0} \limsup _{k \rightarrow \infty} \frac{1}{T}\left|\sum_{i=n_{k}}^{m\left(n_{k}, t\right)} a_{i} o(1)\right|=0
\end{aligned}
$$

where $o(1) \rightarrow 0$ as $i \rightarrow \infty$ because $c_{i} \rightarrow 0$ and $\delta_{i}(\lambda) \rightarrow 0$ as $i \rightarrow \infty$ and $\left\|x_{i}\right\| \leq 2 M$ for all $i \in\left\{n_{k}, \cdots, m\left(n_{k}, t\right)\right\}$. Thus, the noise condition required in Theorem $\mathrm{A}$ in the Appendix is satisfied, while for the function $v$ in Theorem $\mathrm{A},-L$ can be selected (cf. Remark 2). Then sufficiency follows from Theorem A. 
Necessity: Now it is verified that (15) implies (16)-(18). Assume that (15) is satisfied and let $\Omega \backslash \mathcal{N}$ be the set, where $\left(x_{n}, n \in \mathbb{N}\right)$ converges and the series in (38) and (39) are finite for any $N \in \mathbb{N}, P(\mathcal{N})=0$ and fix $\omega \in \Omega \backslash \mathcal{N}$. Again for notational convenience the evaluations at $\omega$ are suppressed. There is a $K \in \mathbb{N}$ such that if $k \geq \sigma_{K}$ then

$$
x_{k+1}=x_{k}+a_{k} y_{k+1} \text {. }
$$

Use the decomposition in [15] so that

$$
\epsilon_{k+1}=\frac{x_{k+1}-x_{k}}{a_{k}}-f\left(x_{k}\right)=\epsilon_{k+1}^{(1)}+\epsilon_{k+1}^{(2)}
$$

where $\epsilon_{k+1}^{(1)}=-f\left(x_{k}\right)$ and $\epsilon_{k+1}^{(2)}=\left(x_{k+1}-x_{k}\right) / a_{k}$. It is clear that

$$
\sum_{k=1}^{\infty} a_{k} \epsilon_{k+1}^{(2)}<\infty
$$

and

$$
\lim _{k \rightarrow \infty} \epsilon_{k+1}^{(1)}=0 .
$$

Since $\left(x_{k}, k \in \mathbb{N}\right)$ is convergent and (38) and (39) are satisfied, it follows that $\lim _{k \rightarrow \infty} w_{k i}^{i}=0, \lim _{k \rightarrow \infty} u_{k i}^{i}=0$ and $\sum_{i=1}^{\infty} a_{i} w_{i \lambda}^{j}<\infty, \sum_{i=1}^{\infty} a_{i} u_{i \lambda}^{j}<\infty$ for all $\lambda \neq j$ and $\lambda$, $j \in\{1, \cdots, l\}$. Thus $\lim _{k \rightarrow \infty} w_{k}(1)=0, \lim _{k \rightarrow \infty} u_{k}(1)=0$ and

$$
\begin{aligned}
& \sum_{i=1}^{\infty} a_{i} w_{i}(2)<\infty \\
& \sum_{i=1}^{\infty} a_{i} u_{i}(2)<\infty
\end{aligned}
$$

where $w_{k}(1), u_{k}(1), w_{k}(2)$, and $u_{k}(2)$ are defined in Lemma 1. From (26) and (52) it follows that

$$
\begin{aligned}
\frac{1}{c_{k}} \xi_{k+1} g_{k}=- & \left.\left(2 f\left(x_{k}\right)+w_{k}(1)\right)+u_{k}(1)\right)+2 \epsilon_{k+1}^{(2)} \\
& \left.-w_{k}(2)-u_{k}(2)\right) .
\end{aligned}
$$

By (35) it follows that

$$
\begin{aligned}
2 f_{i}\left(x_{k}\right)+w_{k i}^{i}+u_{k i}^{i} & \\
= & \frac{L\left(x_{k}+c_{k} \Delta_{k}\right)-L\left(x_{k}+c_{k}\left(\Delta_{k}(i-1)+\Delta_{k}^{c}(i)\right)\right)}{2 c_{k} \Delta_{k}^{i}} \\
& +\frac{L\left(x_{k}-c_{k}\left(\Delta_{k}(i-1)+\Delta_{k}^{c}(i)\right)\right)-L\left(x_{k}-c_{k} \Delta_{k}\right)}{2 c_{k} \Delta_{k}^{i}} .
\end{aligned}
$$

Let $\nu_{k+1}^{i}$ be given by

$$
\begin{aligned}
\nu_{k+1}^{i}= & \frac{1}{2}\left[L\left(x_{k}+c_{k}\left(\Delta_{k}(i-1)+\Delta_{k}^{c}(i)\right)\right)-L\left(x_{k}+c_{k} \Delta_{k}\right)\right. \\
& \left.\left.+L\left(x_{k}-c_{k} \Delta_{k}\right)\right)-L\left(x_{k}-c_{k}\left(\Delta_{k}(i-1)+\Delta_{k}^{c}(i)\right)\right)\right] .
\end{aligned}
$$

By (27) and (28) it follows that

$$
\frac{\nu_{k+1}^{i}}{c_{k} \Delta_{k}^{i}}=-f_{i}\left(x_{k}+c_{k} \Delta_{k}+\delta_{k}(i)\right)-f_{i}\left(x_{k}-c_{k} \Delta_{k}+\delta_{k}(i)\right)
$$

for $i \in\{1, \cdots, l\}$ and

$$
\lim _{k \rightarrow \infty} f_{i}\left(x_{k} \pm c_{k} \Delta_{k}+\delta_{k}(i)\right)=f_{i}\left(x^{0}\right)=0 .
$$

Let $e_{k+1}^{j}$ be given by

$$
e_{k+1}^{j}=\xi_{k+1}-\nu_{k+1}^{j} .
$$

By (57) and (58) it follows that

$$
2 \epsilon_{k+1}^{(2)}-w_{k}(2)-u_{k}(2)=\left[\frac{e_{k+1}^{1}}{c_{k} \Delta_{k}^{1}}, \cdots, \frac{e_{k+1}^{l}}{c_{k} \Delta_{k}^{l}}\right]^{T} .
$$

By (53)-(56) it follows that

$$
\sum_{k=1}^{\infty} a_{k} \frac{e_{k+1}^{j}}{c_{k} \Delta_{k}^{j}}<\infty \text { a.s. }
$$

for $j \in\{1, \cdots, l\}$. Equations (58)-(60) give the required decomposition (16)-(18). The equations (16)-(18) can be verified for the one-sided algorithm in a similar fashion.

Assuming an independence condition the following theorem gives a large family of noise processes that satisfy (16)-(18).

Theorem 2: Let H1) and H2) be satisfied. If $\sum_{k=1}^{\infty}\left(a_{k}^{2} / c_{k}^{2}\right)$ $<\infty$ and the observation noise $\left(\xi_{k}, k \in \mathbb{N}\right)$ has the property that $\xi(k+1)$ is independent of $\left(\Delta_{j}, j=1,2, \cdots, k\right)$ for each $k \in \mathbb{N}$ and satisfies one of the following two conditions:

1) $\sup _{k}\left|\xi_{k}\right| \leq \xi$ a.s. where $\xi$ is a random variable;

2) $\sup _{k} E \xi_{k}^{2}<\infty$;

then

$$
\lim _{k \rightarrow \infty} x_{k}=x^{0} \quad \text { a.s. }
$$

where $x_{k}$ is given by (13).

Proof: Initially it should be noted that $\xi_{k}$ may depend arbitrarily on $\left(\xi_{j}, j \neq k\right)$ and may not be zero mean, e.g., a sequence of bounded deterministic observation errors satisfies the conditions.

It is only necessary to verify (16)-(18). Let 1) be satisfied, that is, there is a random variable $\xi$ such that $\sup _{k \in \mathbb{N}}\left|\xi_{k}\right| \leq$ $\xi<\infty$. For $j \in\{1, \cdots, \ell\}$ and $k \in \mathbb{N}$ let $\mathcal{F}_{k}^{j}$ be given by

$$
\mathcal{F}_{k}^{j}=\sigma\left(\Delta_{i}^{j}, i \in\{0, \cdots, k\}, \xi_{p}, p \in\{0, \cdots, k+2\}\right) .
$$

By the definition of $\Delta_{k}$, it is independent of $\xi_{k+1}$ and $\mathcal{F}_{k-1}^{j}$ so that

$$
E\left[\frac{\xi_{k+1}}{\Delta_{k}^{j}} \mid \mathcal{F}_{k-1}^{j}\right]=\xi_{k+1} E\left[\frac{1}{\Delta_{k}^{j}} \mid \mathcal{F}_{k-1}^{j}\right]=0 \text { a.s. }
$$

and

$$
\sum_{k=1}^{\infty} E\left[\frac{a_{k}^{2}}{c_{k}^{2}} \frac{\xi_{k+1}^{2}}{\left(\Delta_{k}^{j}\right)^{2}} \mid \mathcal{F}_{k-1}^{j}\right] \leq \sum_{k=1}^{\infty} \frac{a_{k}^{2}}{c_{k}^{2}} b^{2} \xi^{2}<\infty \quad \text { a.s. }
$$

By the convergence theorem for mds's [3], [6], it follows that

$$
\sum_{k=1}^{\infty} \frac{a_{k}}{c_{k}} \frac{\xi_{k+1}}{\Delta_{k}^{j}}<\infty \quad \text { a.s. }
$$

for all $j \in\{1, \cdots, \ell\}$. Thus in (16) it can be assumed that $\nu_{k}^{j} \equiv 0$ and $e_{k}^{j}=\xi_{k}$ for $j=1, \cdots, \ell$.

Now assume that $\sup _{k \in \mathbb{N}} E \xi_{k}^{2}<\infty$ is satisfied. 
By the independence assumption it follows that for $j<k$, and $\Delta_{k}$ is independent of $\left(\Delta_{k}, \xi_{k+1}, \xi_{j+1}\right)$ so that

$$
E\left[\frac{\xi_{j+1} \xi_{k+1}}{\Delta_{j}^{i} \Delta_{k}^{i}}\right]=E\left[\frac{\xi_{j+1} \xi_{k+1}}{\Delta_{k}^{i}}\right] E\left[\frac{1}{\Delta_{j}^{i}}\right]=0
$$

for all $i \in\{1, \cdots, \ell\}$ so that

$$
E\left[\left(\sum_{k=1}^{\infty} \frac{a_{k}}{c_{k}} \frac{\xi_{k+1}}{\Delta_{k}^{j}}\right)^{2}\right] \leq b^{2} \sup _{k \in s} E \xi_{k+1}^{2} \sum_{k=1}^{\infty} \frac{a_{k}^{2}}{c_{k}^{2}}<\infty
$$

where $b$ is an upper bound of $\left|1 / \Delta_{k}^{i}\right|$. It follows directly that

$$
\sum_{k=1}^{\infty} \frac{a_{k}}{c_{k}} \frac{\xi_{k+1}}{\Delta_{k}^{j}}<\infty \quad \text { a.s. }
$$

for all $j=1, \cdots, \ell$. To verify (17) and (18) it suffices to choose $\nu_{k}^{j} \equiv 0$ and $e_{k}^{j}=\xi_{k}$.

Remark 2: Using Theorem A in the Appendix, Theorems 1 and 2 can be extended to the case where $f(x)=0$ for all $x \in J$ and $J$ is not a singleton. In this case H1) is replaced by $\mathrm{C} 22$ ) and 3) in Theorem A where $v=-L$ and $f$ is locally Lipschitz continuous.

\section{RATE OF CONVERGENCE AND ASYMPTOTIC NORMALITY}

In the following theorem a rate of convergence of the algorithm (13) is given.

Theorem 3: Assume the hypotheses of Theorem 2 and that

$$
\begin{aligned}
\lim _{n \rightarrow \infty}\left(a_{n+1}^{-1}-a_{n}^{-1}\right) & =\alpha \geq 0 \\
c_{k} & =o\left(a_{k}^{\delta}\right) \\
\sum_{j=1}^{\infty} a_{j}^{2(1-\delta)} / c_{j}^{2} & <\infty
\end{aligned}
$$

for some $\delta \in(0,1)$ and

$$
f(x)=F\left(x-x^{0}\right)+\gamma(x)
$$

where $F \in L\left(\mathbb{R}^{\ell}, \mathbb{R}^{\ell}\right), \gamma(x)=o\left(\left\|x-x^{0}\right\|\right)$ and $F+\alpha \gamma I$ is stable. Then $\left(x_{n}, n \in \mathbb{N}\right)$ given by (13) satisfies

$$
\left\|x_{n}-x^{0}\right\|=o\left(a_{n}^{\delta}\right) \quad \text { a.s. }
$$

for $\delta$ given in (63).

Proof: By Theorem B in the Appendix it suffices to show that $\left(\varepsilon_{n}, n \in \mathbb{N}\right)$ given by (25) or (26) can be represented as $\varepsilon_{n}=e_{n}+\nu_{n}$ where $\nu_{n}=o\left(a_{n}^{\delta}\right)$ as $n \rightarrow \infty$ and $\sum_{k=1}^{\infty} a_{k}^{1-\delta} e_{k+1}<\infty$ a.s. It suffices to verify this result for (25), because the proof for (26) is similar. It follows by Theorem 2 that $\lim _{n \rightarrow \infty} x_{n}=x^{0}$ a.s. By the local Lipschitz condition on $f$ it follows from (31) that

$$
\left\|w_{k}(1)\right\|=O\left(c_{k}\right)=o\left(a_{k}^{\delta}\right)
$$

as $k \rightarrow \infty$. Since $c_{k}=o\left(a_{k}^{\delta}\right)$ it follows that

$$
a_{k}^{1-\delta}=o\left(\frac{a_{k}}{c_{k}}\right)
$$

$$
\sum_{k=1}^{\infty} a_{k}^{2(1-\delta)}<\infty
$$

It follows that $\left|h_{k j}(\omega)\right|$ in (38) is uniformly bounded for $j \in\{1, \cdots, \ell\}$ and $k \in \mathbb{N}$ for each $\omega$ where $\left(x_{n}, n \in \mathbb{N}\right)$ converges to $x^{0}$. By the convergence theorem for mds's it follows that

$$
\sum_{j=1}^{\infty} a_{j}^{1-\delta} w_{j}(2)<\infty \quad \text { a.s. }
$$

As in the proof of Theorem 2 it follows from (63) that

$$
\sum_{j=1}^{\infty} a_{j}^{1-\delta} \frac{\xi_{j+1}}{c_{j}} g_{j}<\infty \quad \text { a.s. }
$$

Using (66) and the last two inequalities verifies (65).

Remark 3: If $a_{n}=1 / n$ and $c_{n}=1 / n^{v}$ for some $v \in$ $(0,1 / 2)$ and all $n \in \mathbb{N}$, then the conditions on $\left(a_{n}, n \in \mathbb{N}\right)$ and $\left(c_{n}, n \in \mathbb{N}\right)$ in Theorem 3 are satisfied.

The following result is an asymptotic normality property of $\left(x_{n}, n \in \mathbb{N}\right)$ given by the algorithm (13).

Theorem 4: Assume that the conditions H1) and $\mathrm{H} 2$ ) are satisfied and that:

1) $\lim _{n \rightarrow \infty}\left(a_{n+1}^{-1}-a_{n}^{-1}\right)=\alpha>0$ and $c_{n}=a_{n}^{\gamma}$ for some $\gamma \in(1 / 4,1 / 2)$

2) $\left\|f(x)-F\left(x-x^{0}\right)\right\| \leq b\left\|x-x^{0}\right\|^{1+\beta}$ for some $\beta>0$ and $b>0$

3) $F+\alpha \gamma I$ is stable and $\sum_{j=1}^{\infty} a_{j}^{2(1-\gamma-\delta)}<\infty$ for some $\delta \in(\gamma /(1+\beta), \gamma)$

4) $\xi_{n}=\sum_{i=0}^{r} b_{i} w_{n-i}$ if $\left\{y_{n}\right\}$ is given by (12), and $\xi_{n}=2 \sum_{i=0}^{r} b_{i} w_{n-i}$ if $\left\{y_{n}\right\}$ is given by $\left(12^{\prime}\right)$ where $w_{i}=0$ for $i<0,\left(b_{i}, i \in\{0,1, \cdots, r\}\right)$ is a sequence of real numbers, $r \in \mathbb{N}$ is fixed and $\left(w_{i}, \mathcal{F}_{i}^{w}, i \in \mathbb{N}\right)$ is an mds that is independent of $\left(\Delta_{i}, i \in \mathbb{N}\right)$ and satisfies

$$
E\left[w_{i}^{2} \mid \mathcal{F}_{i-1}^{w}\right] \leq \sigma_{0}
$$

for all $i \in \mathbb{N}$ where $\sigma_{0} \in \mathbb{R}_{+}$

$$
\lim _{i \rightarrow \infty} E\left[w_{i}^{2} \mid \mathcal{F}_{i-1}^{w}\right]=\sigma^{2}
$$

where $\sigma^{2} \in \mathbb{R}_{+}$and

$$
\lim _{N \rightarrow \infty} \sup _{i \in \mathbb{N}} E\left[w_{i}^{2} 1_{\left\{\left|w_{i}\right|>N\right\}}\right]=0 .
$$

Then for both the one-sided and the two-sided algorithms

$$
a_{n}^{-\mu}\left(x_{n}-x_{0}\right) \stackrel{d}{\rightarrow} Z
$$

where $\mu=(1 / 2)-\gamma$ and $Z$ is an $N(0, S)$ random variable

$$
\begin{aligned}
S & =\sigma^{2} \sigma_{\Delta}^{2}\left(\sum_{i=0}^{r} b_{i}\right)^{2} \int_{0}^{\infty} e^{t(F+\alpha \mu I)} e^{t(F+\alpha \mu I)^{T}} d t \\
\sigma_{\Delta}^{2} & =E\left[\frac{1}{\left(\Delta_{i}^{1}\right)^{2}}\right]
\end{aligned}
$$


Proof: Since $\gamma>\delta$, it follows that $c_{k}=a_{k}^{\gamma}=o\left(a_{k}^{\delta}\right)$, $\sum_{j=1}^{\infty} a_{j}^{2(1-\delta)} / c_{j}^{2}=\sum_{j=1}^{\infty} a_{j}^{2(1-\gamma-\delta)}<\infty$ and by Theorem 3

$$
\left\|x_{n}-x^{0}\right\|=o\left(a_{n}^{\mu}\right)
$$

and after a finite number of iterations of (13) there are no subsequent truncations.

Since $\mu=(1 / 2)-\gamma, \gamma \in(1 / 4,1 / 2), 0<\mu<1 / 4, \gamma>\mu$, and $F+\alpha \mu I$ is stable, it follows directly that

$$
\begin{aligned}
\frac{a_{n+1}^{-\mu}}{a_{n}^{-\mu}} & =\left(1+\frac{a_{n+1}^{-1}-a_{n}^{-1}}{a_{n}^{-1}}\right)^{\mu} \\
& =\left(1+\alpha a_{n}+a_{n}\left(a_{n+1}^{-1}-a_{n}^{-1}-\alpha\right)\right)^{\mu} \\
& =1+\alpha \mu a_{n}+o\left(a_{n}\right) .
\end{aligned}
$$

Let $u_{n}$ be given by

$$
u_{n}=a_{n}^{-\mu}\left(x_{n}-x^{0}\right)
$$

so that

$$
\begin{aligned}
u_{n+1}= & \left(1+\alpha \mu a_{n}+o\left(a_{n}\right)\right) \times\left(u_{n}+a_{n} a_{n}^{-\mu}\right. \\
& \times\left(F\left(x_{n}-x^{0}\right)+f\left(x_{n}\right)-F\left(x-x^{0}\right)\right. \\
& \left.\left.+w_{n}(1)+w_{n}(2)+\frac{1}{c_{n}} \xi_{n+1} g_{n}\right)\right) \\
= & {\left[I+a_{n}(F+\alpha \mu I+o(1))\right] u_{n} } \\
& +\left(1+O\left(a_{n}\right)\right) a_{n}^{(1 / 2)+\gamma} b\left\|x_{n}-x^{0}\right\|^{1+\beta} \\
& +\left(1+O\left(a_{n}\right)\right) a_{n}^{(1 / 2)+\gamma}\left(w_{n}(1)+w_{n}(2)\right) \\
& +\left(1+O\left(a_{n}\right)\right) a_{n}^{(1 / 2)} \xi_{n+1} g_{n}
\end{aligned}
$$

where subsequently the verification is made only for (12) because for $\left(12^{\prime}\right)$ it is only necessary to replace $\xi_{n+1}$ by $\xi_{n+1} / 2$, and $w_{n}(1), w_{n}(2)$, and $g_{n}$ are given in Lemma 1 .

Let $\Phi_{n k}$ be given by

$$
\begin{aligned}
\Phi_{n k} & =\prod_{i=k}^{n}\left(I+a_{i}(F+\alpha \mu I)+o(1)\right) \\
\Phi_{j, j+1} & =I
\end{aligned}
$$

where $k<n$. Since $F+\alpha \mu I$ is stable it follows that for $n$ large

$$
\left\|\Phi_{n k}\right\| \leq \lambda_{1} \exp \left(-\lambda \sum_{i=k}^{n} a_{i}\right)
$$

where $\lambda_{1}$ and $\lambda$ are strictly positive constants.

Consider the following algorithm:

$$
\begin{aligned}
\hat{\eta}_{n+1} & =\left(I+a_{n}(F+\alpha \mu I+o(1))\right) \eta_{n}+a_{n}^{(1 / 2)+\gamma} w_{n}(2) \\
\eta_{n+1} & =\hat{\eta}_{n+1} 1_{\left\{\left\|\hat{\eta}_{n+1}\right\| \leq M_{\sigma_{n}}\right\}}+\eta^{*} 1_{\left\{\left\|\hat{\eta}_{n+1}\right\|>M_{\sigma_{n}}\right\}} \\
\sigma_{n} & =\sum_{i=0}^{n-1} 1_{\left\{\left\|\hat{\eta}_{i}\right\|>M_{\sigma_{n}}\right\}}
\end{aligned}
$$

where $M_{k}>0$ for all $k \in \mathbb{N}$ and $M_{k} \uparrow \infty$ as $k \rightarrow \infty$. Since $\sum_{k=1}^{\infty} a_{k}^{2-2 \gamma}<\infty$ and $\gamma>1 / 4, \sum_{k=1}^{\infty} a_{k}^{1+2 \gamma}<\infty$. By the martingale convergence theorem $\sum_{k=1}^{\infty} a_{k}^{(1 / 2)+\gamma} w_{k}(2)<\infty$ a.s.

Similar to the proof of Lemma 2, it can be verified that for any convergent subsequence $\left\{\eta_{n_{k}}\right\}$ there are positive constants
$M, T$, and $k_{T}$ such that if $k>k_{T}$, then

$$
\left\|\sum_{i=n_{k}}^{m+1} a_{i}\left[(F+\alpha \mu I+o(1)) \eta_{i}+a_{i}^{\gamma-(1 / 2)} w_{i}(2)\right]\right\| \leq M .
$$

Therefore, for any fixed $\omega$ there is $c(\omega)$ such that $\left\|\eta_{i}(\omega)\right\| \leq$ $c(\omega), \forall i: n_{k} \leq i \leq m\left(n_{k}, T\right)$, and

$$
\lim _{T \rightarrow 0} \limsup _{k \rightarrow \infty} \frac{1}{T}\left\|\sum_{i=n_{k}}^{m\left(n_{k}, t\right)}\left(a_{i} o(1) \eta_{i}+a_{i}^{(1 / 2)+\gamma} w_{i}(2)\right)\right\|=0 .
$$

Since $F+\alpha \mu I$ is stable, by Theorem A, $\eta_{n} \rightarrow 0$ a.s. as $n \rightarrow \infty$. Therefore, the algorithm for $\eta_{n}$ becomes the one without truncations starting from some $n_{0}$, i.e.,

$$
\eta_{n+1}=\left(I+a_{n}(F+\alpha \mu I+o(1)) \eta_{n}+a_{n}^{(1 / 2)+\gamma} w_{n}(2)\right.
$$

for $n \geq n_{0}$ and hence

$$
\eta_{n+1}=\Phi_{n, n_{0}} \eta_{n_{0}}+\sum_{i=n_{0}}^{n} \Phi_{n, i+1} a_{i}^{(1 / 2)+\gamma} w_{i}(2) .
$$

By (71) and the convergence of $\left(\eta_{n}, n \in \mathbb{N}\right)$ to zero it follows that

$$
\sum_{i=n_{0}}^{n} \Phi_{n, i+1} a_{i}^{(1 / 2)+\gamma}(2) \rightarrow 0, \quad \text { a.s. as } n \rightarrow \infty
$$

and hence $\sum_{i=1}^{n} \Phi_{n, i+1} a_{i}^{(1 / 2)+\gamma} w_{i}(2) \rightarrow 0$ a.s. as $n \rightarrow \infty$, which implies

$$
\lim _{n \rightarrow \infty} \sum_{i=1}^{n} \Phi_{n, i+1}\left(1+O\left(a_{i}\right)\right) a_{i}^{(1 / 2)+\gamma} w_{i}(2)=0 \quad \text { a.s. }
$$

By (69) it follows that

$$
\begin{aligned}
(1 & \left.+O\left(a_{n}\right)\right) a_{n}^{(1 / 2)+\gamma} b\left\|x_{n}-x^{0}\right\|^{1+\beta} \\
\quad & \leq b\left(1+O\left(a_{n}\right)\right) a_{n}^{(1 / 2)+\gamma} a_{n}^{(1+\beta) \delta}=O\left(a_{n}^{1+\epsilon}\right)
\end{aligned}
$$

where $\epsilon \triangleq(1+\beta) \delta+(1 / 2)+\gamma>(1 / 2)+2 \gamma \triangleq \epsilon^{\prime}>0$, and from (27), it follows that

$$
\left(1+O\left(a_{n}\right)\right) a_{n}^{(1 / 2)+\gamma} w_{n}(1)=O\left(a_{n}^{(1 / 2)+\gamma} O\left(c_{n}\right)\right)=O\left(a_{n}^{1+\epsilon^{\prime}}\right) .
$$

Solving the recursion (70) it follows that

$$
\begin{aligned}
u_{n+1}= & \Phi_{n 1} u_{1}+\sum_{i=1}^{n} \Phi_{n i+1} o\left(a_{i}\right) \\
& +\sum_{i=1}^{n} \Phi_{n, i+1}\left(1+O\left(a_{i}\right)\right) a_{i}^{(1 / 2)+\gamma} w_{i}(2) \\
& +\sum_{i=1}^{n} \Phi_{n i+1} a_{i}^{1 / 2} \xi_{i+1} g_{i} .
\end{aligned}
$$




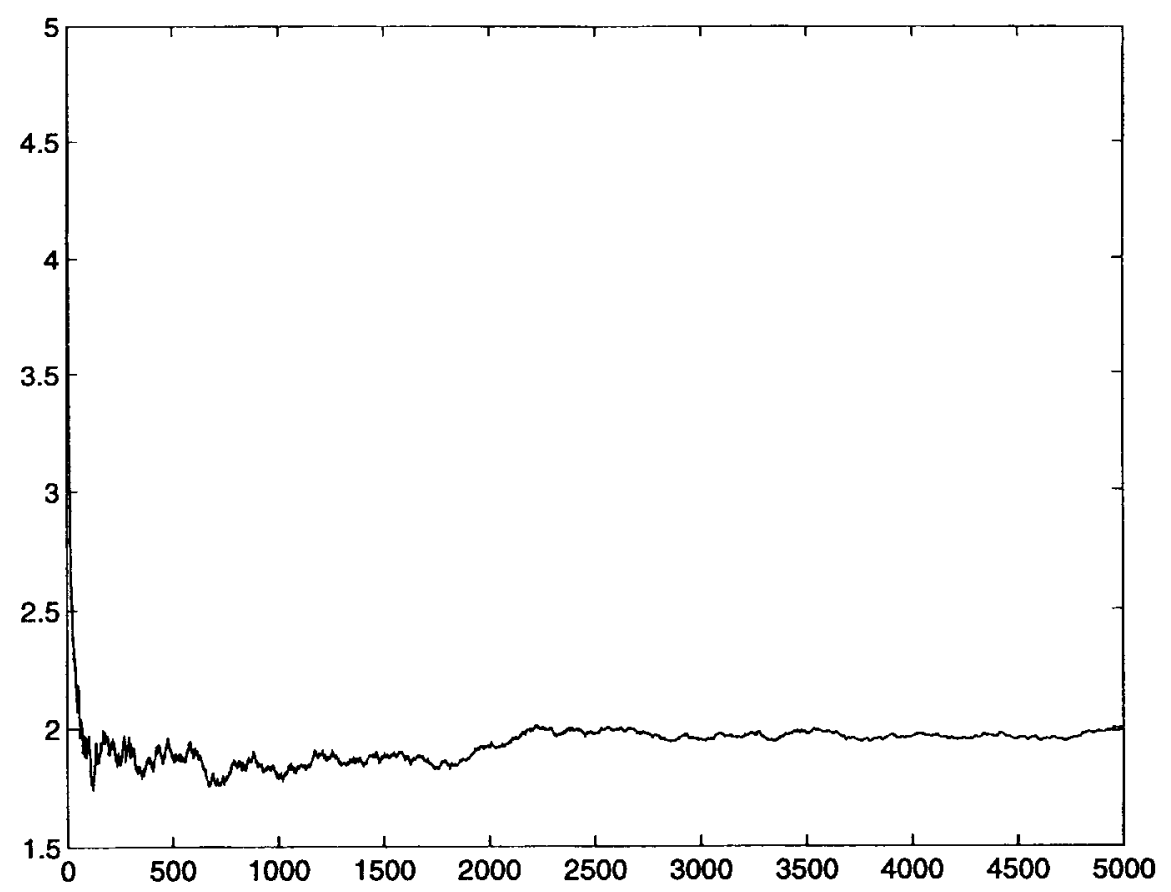

Fig. 1. The path of $x_{k}$ with $x^{*}=5$ and initial value $x_{0}=3$.

Since

$$
\begin{aligned}
\sum_{i=1}^{n} & a_{i} \exp \left(-\lambda \sum_{j=i+1}^{n} a_{j}\right) \\
\leq & \frac{1}{\lambda} \sum_{i=1}^{n}\left(1-e^{-\lambda a_{i}}+\frac{\left(\lambda a_{i}\right)^{2}}{2}\right) \exp \left(-\lambda \sum_{j=i+1}^{n} a_{j}\right) \\
= & \frac{1}{\lambda} \sum_{i=1}^{n}\left(\exp \left(-\lambda \sum_{j=i+1}^{n} a_{j}\right)-\exp \left(-\lambda \sum_{j=i}^{n} a_{j}\right)\right) \\
& +\frac{\lambda}{2} \sum_{i=1}^{n} a_{i}^{2} \exp \left(-\lambda \sum_{j=i+1}^{n} a_{j}\right)
\end{aligned}
$$

it follows that

$$
\sum_{i=1}^{n}\left(1-\frac{\lambda a_{i}}{2}\right) a_{i} \exp \left(-\lambda \sum_{j=i+1}^{n} a_{j}\right) \leq \frac{1}{\lambda} .
$$

Since $a_{n} \rightarrow 0$ as $n \rightarrow \infty$ it follows that

$$
\sum_{i=1}^{n} a_{i} \exp \left(-\lambda \sum_{j=i+1}^{n} a_{j}\right)
$$

is uniformly bounded for $n \in \mathbb{N}$. This boundedness and (71)

imply that

$$
\lim _{n \rightarrow \infty} \sum_{i=1}^{n} \Phi_{n, i+1} \cdot\left(a_{i}\right)=0 .
$$

Thus

$$
\lim _{n \rightarrow \infty}\left(u_{n+1}-\sum_{i=1}^{n} \Phi_{n, i+1} a_{i}^{1 / 2} \xi_{i+1} g_{i}\right)=0 \quad \text { a.s. }
$$

Since $\left(\Delta_{n}, n \in \mathbb{N}\right)$ is independent of $\left(\xi_{n}, n \in \mathbb{N}\right)$ it follows by a standard method [5], [8], which is sketched in the Appendix, that

$$
\sum_{i=1}^{n} \Phi_{n, i+1} a_{i}^{1 / 2} \xi_{i+1} g_{i} \stackrel{d}{\rightarrow} Z
$$

where $Z$ is $N(0, S)$.

\section{A Numerical Example}

An elementary numerical example is given of the onesided algorithm (10), (13). Let $L(x)=-x^{2}+4 x+2$, which has a unique maximum at $x=2$. The random variable $\Delta_{k}^{i}$ is uniformly distributed on $[-1,-0.5] \cup[0.5,1]$ for $i \in\{1, \cdots, \ell\}$. The noise processes $\left(\xi_{k}^{+}, k \in \mathbb{N}\right)$ and $\left(\xi_{k}^{0}\right.$, $k \in \mathbb{N}$ ) are independent white Gaussian processes and the constants $a_{k}, c_{k}$, and $M_{k}$ are $a_{k}=1 /(k+20), c_{k}=1 / k^{1 / 5}$, and $M_{k}=2 k$ for $k \in \mathbb{N}$. In Fig. 1 is a graph of $\left\{x_{k}\right.$, $0 \leq k \leq 5000\}$ with $x_{0}=3$ and $x^{*}=5$. In Figs. 2 and 3 are graphs of $\left(y_{k}^{+}, 0 \leq k \leq 5000\right)$ and $\left(y_{k}^{0}, 0 \leq k \leq 5000\right)$, respectively.

\section{CONCLUSION}

The classical Kiefer-Wolfowitz algorithm has been modified in two ways: 1) the one-sided and the two-sided randomized differences are used instead of the two-sided deterministic differences and 2) the estimates are truncated at randomly varying bounds. For the convergence analysis, a direct method is used rather than the classical probabilistic method or the ODE method. By the algorithm modifications 1) and 2) and a different approach to algorithm analysis, the following algorithm improvements have been made: 1) some restrictive 


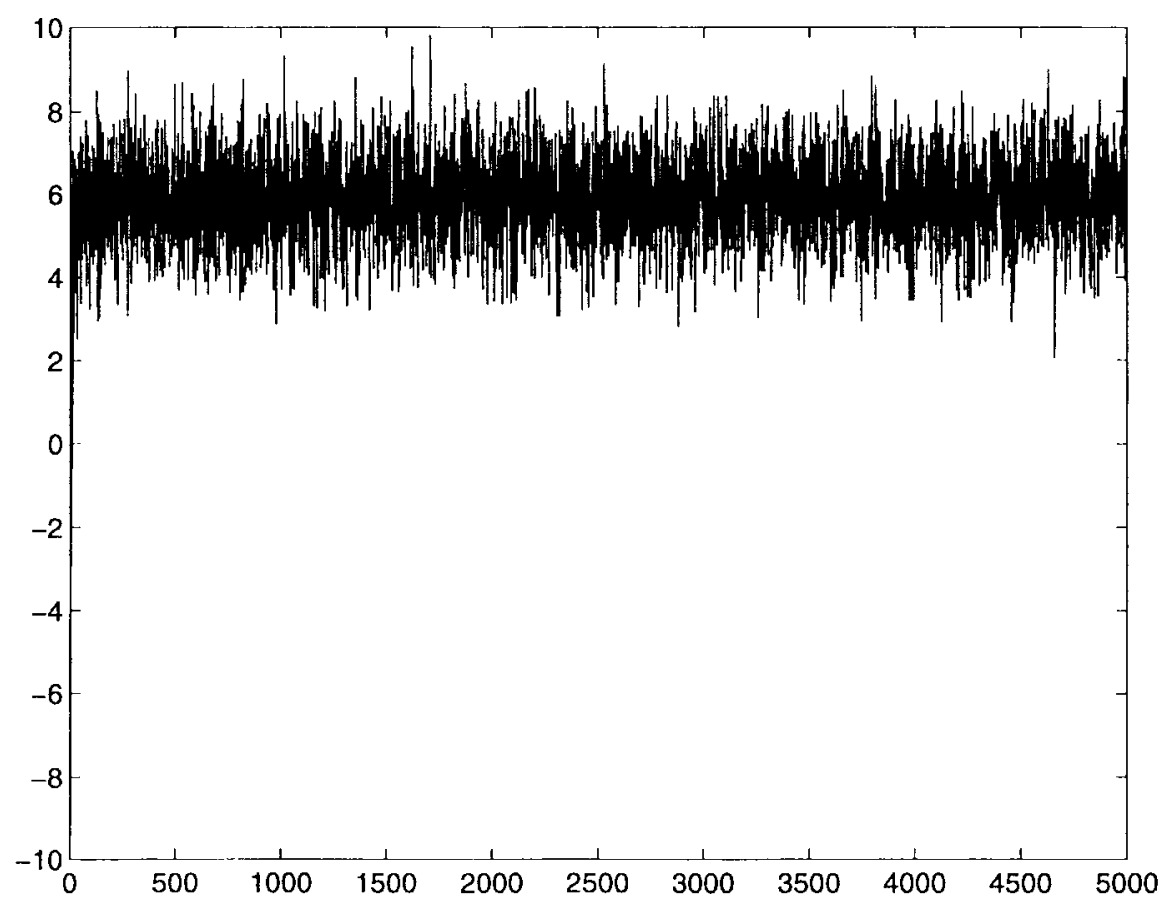

Fig. 2. The path of the observation $y_{k+1}^{+}$.

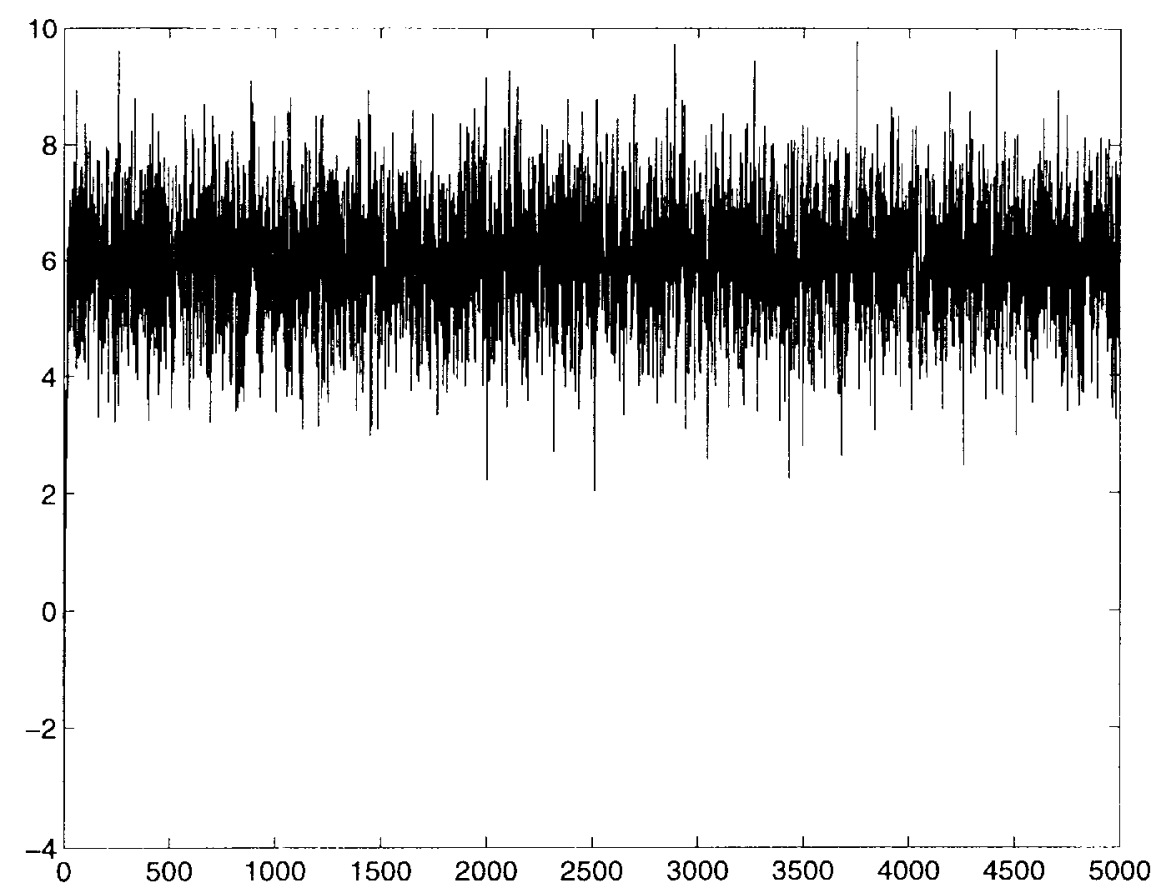

Fig. 3. The path of the observation $y_{k+1}^{0}$.

conditions on the function $L$ or some boundedness assumptions on the estimates have been removed; 2) some restrictive conditions on the noise process have been removed; and 3) the number of required observations at each iteration have been reduced. If the function $L$ has many extrema then the algorithm may become stuck at a local extremum. To obtain the almost sure convergence to global extrema, some methods that combine search and stochastic approximation are needed, but it seems that there is a lack of a complete theory for this approach.

\section{APPENDIX}

Theorem A: Assume that the following conditions are satisfied.

C0) The function $f: \mathbb{R}^{\ell} \rightarrow \mathbb{R}$ is Borel measurable and locally bounded and $J=\{x: f(x)=0\}$.

C1) The sequence of strictly positive numbers $\left(a_{n}, n \in \mathbb{N}\right)$ converges to zero and $\sum_{n=1}^{\infty} a_{n}=\infty$.

C2) There is a continuously differentiable function $v$ : $\mathbb{R}^{\ell} \rightarrow \mathbb{R}$ such that 
1) $\sup _{\delta \leq d(x, J) \leq \Delta} f^{T}(x) v_{x}(x)<0$ for all $\Delta>\delta>$ 0 where $v_{x}$ is the gradient of $v$ and $d(x, S)=$ $\inf \{\|x-y\|: y \in S\}$.

2) $v(J)$ is nowhere dense.

3) There is a $c_{0}>0$ such that $\left\|x^{*}\right\|<c_{0}$

$$
v\left(x^{*}\right)<\inf _{\|x\|=c_{0}} v(x) \text {. }
$$

Then $\left(x_{n}, n \in \mathbb{N}\right)$ defined by (13) with

$$
y_{n+1}=f\left(x_{n}\right)+\varepsilon_{n+1}
$$

converges to the set $J$ on $A$ where

$$
A=\left\{\lim _{T \rightarrow 0} \limsup _{k \rightarrow \infty} \frac{1}{T}\left\|\sum_{i=n_{k}}^{m\left(n_{k}, t\right)} a_{i} \varepsilon_{i+1}\right\|=0\right.
$$

for all $t \in[0, T]$ whenever $\left(n_{k}, k \in \mathbb{N}\right)$ is such that $\left(x_{n_{k}}, k \in \mathbb{N}\right)$ is convergent $\}$

and

$$
m(k, T)=\max \left\{m: \sum_{i=k}^{m} a_{i} \leq T\right\} .
$$

The proof of this result is given in [1] or [2].

Remark A1: Given the conditions of Theorem A, $\left(x_{n}, n \in\right.$ IN) converges so that

$$
\begin{aligned}
A= & A^{\prime} \\
= & \left\{\lim _{T \rightarrow 0} \limsup _{k \rightarrow \infty} \frac{1}{T}\left\|\sum_{i=k}^{m(k, T)} a_{i} \varepsilon_{i+1}\right\|=0\right. \\
& \text { for all } t \in[0, T]\} .
\end{aligned}
$$

However, it should be noted that the equality $A=A^{\prime}$ is satisfied only with the conditions $\mathrm{C} 0)-\mathrm{C} 2$ ). Without these conditions there is only the inclusion $A^{\prime} \subset A$. If $\varepsilon_{i+1}$ depends on $x_{i}$ then since the behavior of $\left(x_{n}, n \in \mathbb{N}\right)$ is not known it is difficult to verify if a point is in $A^{\prime}$ while to verify a point is in $A$ is easier because only convergent subsequences need to be considered.

Remark A2: If $J=\left\{x^{0}\right\}$ then C2-2) is immediately satisfied and C2-3) becomes $\left\|x^{*}\right\|<c_{0}$ and $v\left(x^{*}\right)<$ $\inf _{\|x\|=c_{0}} v(x)$ for some $c_{0}$.

Theorem B: If the conditions $\mathrm{C}($ ) $-\mathrm{C} 2$ ) of Theorem A are satisfied where $J=\left\{x^{0}\right\}$ and

$$
\lim _{n \rightarrow \infty}\left(a_{n+1}^{-1}-a_{n}^{-1}\right)=\alpha \geq 0
$$

$\varepsilon_{n}=e_{n}+\nu_{n}, \nu_{n}=o\left(a_{n}^{\gamma}\right), \sum_{i=1}^{\infty} a_{i}^{1-\gamma} e_{i+1}<\infty, H+\alpha \gamma I$ is stable for some $\delta \in(0,1]$ and as $x \rightarrow x^{0}, f(x)=$ $H\left(x-x^{0}\right)+\delta(x), \delta\left(x^{0}\right)=0$, and $\delta(x)=o\left(\left\|x-x^{0}\right\|\right)$ then

$$
\left\|x_{n}-x^{0}\right\|=o\left(a_{n}^{\delta}\right)
$$

as $n \rightarrow \infty$ where $\left(x_{n}, n \in \mathbb{N}\right)$ is given by (13) and $y_{n+1}$ is given by (71).
The proof of this theorem is given in [4].

A sketch of the proof that $\sum_{i=1}^{n} \Phi_{n, i+1} a_{i}^{1 / 2} \xi_{i+1} g_{i} \stackrel{d}{\rightarrow} Z$ is given. Let $\Psi_{n, k}=\prod_{i=k}^{n}\left(I+a_{i}(F+\alpha \mu I)\right), \Psi_{j, j+1}=I$.

Then

$$
\begin{aligned}
\lim _{n \rightarrow \infty} & \sum_{i=1}^{n} \Phi_{n, i+1} a_{i}^{1 / 2} \xi_{i+1} g_{i} \\
- & \sum_{i=1}^{n} \Psi_{n, i+1} a_{i}^{1 / 2} \xi_{i+1} g_{i}=0 \quad \text { a.s. }
\end{aligned}
$$

Therefore, it suffices to show that

$$
\sum_{i=1}^{n} \Psi_{n, i+1} a_{i}^{1 / 2} \xi_{i+1} g_{i} \stackrel{d}{\rightarrow} N(0, S)
$$

By using the Central Limit theorem for random vectors with two indices in [13], it is straightforward to verify that all of the conditions required for $\xi_{n, i+1} \triangleq \Psi_{n, i+1} a_{i}^{1 / 2}$.

$\underset{\text { Hence }}{\left(\sum_{j=0}^{r} b_{j} g_{i+j}\right) w_{i+1} \text { are satisfied. }}$

$$
\sum_{i=1}^{n} \Psi_{n, i+1} a_{i}^{1 / 2}\left(\sum_{j=0}^{r} b_{j} g_{i+j}\right) w_{i+1} \stackrel{d}{\rightarrow} N(0, S) .
$$

It now remains to show that

$$
\begin{aligned}
\lim _{n \rightarrow \infty} \eta_{n} \triangleq & \sum_{i=1}^{n} \Psi_{n, i+1} a_{i}^{1 / 2}\left(\sum_{j=0}^{r} b_{j} w_{i+1-j} g_{i}\right) \\
& -\sum_{i=1}^{n} \Psi_{n, i+1} a_{i}^{1 / 2}\left(\sum_{j=0}^{r} b_{j} g_{i+j}\right) w_{i+1}=0
\end{aligned}
$$

in probability.

Write $\eta_{n}$ as

$$
\begin{aligned}
\eta_{n}= & \sum_{j=0}^{r} \sum_{i=1}^{j} \Psi_{n, i+1} a_{i}^{1 / 2} b_{j} w_{i+1-j} g_{i} \\
& -\sum_{j=0}^{r} \sum_{i=n-j+1}^{n} \Psi_{n, i+1} a_{i}^{1 / 2} b_{j} w_{i+1} g_{i+j} \\
& -\sum_{j=0}^{r} \sum_{i=1}^{n-j}\left(a_{i}^{1 / 2} \Psi_{n, i+1}-a_{i+j}^{1 / 2} \Psi_{n, i+j+1}\right) b_{j} w_{i+1} g_{i+j} .
\end{aligned}
$$

By (71) the first term on the right-hand side of $\eta_{n}$ tends to zero a.s., while the second term tends to zero in probability since the expectation of its norm converges to zero as $n \rightarrow \infty$.

The last term in the expression of $\eta_{n}$ equals

$$
\begin{aligned}
& \sum_{j=0}^{r} \sum_{i=1}^{n-j} a_{i}^{1 / 2} \sum_{k=1}^{j} \Psi_{n, i+k+1} a_{i+k}(F+\alpha \mu I) b_{j} w_{i+1} g_{i+j} \\
& \quad+\sum_{j=0}^{r} \sum_{i=1}^{n-j}\left(\left(\frac{a_{i}}{a_{i+j}}\right)^{1 / 2}-1\right) a_{i+j}^{1 / 2} \Psi_{n, i+j+1} b_{j} w_{i+1} g_{i+j} .
\end{aligned}
$$

Taking expectation of the norm of each term and noting that $\sup _{n} \sum_{k=1}^{n} a_{k}\left\|\Psi_{n, k+1}\right\|^{r}<\infty, \forall r>0$, and $\left(a_{n} / a_{n+1}\right)^{1 / 2}=1+(1 / 2) \alpha a_{n}+o\left(a_{n}\right)$, it is concluded that both terms converge to zero in probability. Therefore, $\eta_{n} \rightarrow 0$ in probability as $n \rightarrow \infty$. 


\section{ACKNOWLEDGMENT}

The authors would like to thank J. Spall and the referees for their useful comments.

\section{REFERENCES}

[1] H. F. Chen, "Stochastic approximation and its new applications," in Proc. Hong Kong Int. Workshop on New Directions of Control and Manufacturing, 1994, pp. 2-12.

[2] H. F. Chen, T. E. Duncan, and B. Pasik-Duncan, "On the ODE approach to parameter identification and its application to adaptive control," to be published.

[3] H. F. Chen and L. Guo, Identification and Stochastic Adaptive Control. Boston, MA: Birkhauser, 1991.

[4] H. F. Chen and Y. M. Zhu, "Asymptotical properties of randomly truncated stochastic approximation procedures," Acta Math. Scientia, vol. 7, pp. 431-441, 1986.

[5] Stochastic Approximation. Shanghai Sci., 1996.

[6] Y. S. Chow, "Local convergence of martingales and the law of large numbers," Ann. Math. Stat., vol. 36, pp. 552-558, 1965.

[7] V. Fabian, "Stochastic approximation," in Optimizing Methods in Statistics, J. S. Rustagi, Ed. New York: Academic, 1971, pp. 439-470.

[8] P. Hall and C. C. Heude, Martingale Limit Theory and Its Applications. New York: Academic, 1980.

[9] J. Kiefer and J. Wolfowitz, "Stochastic approximation of a regression function," Ann. Math. Stat., vol. 23, pp. 462-466, 1952

[10] J. Koronacki, "Random-seeking methods for the stochastic unconstrained optimization," Int. J. Contr., vol. 21, pp. 517-527, 1975.

[11] _ _ "Some remarks on stochastic approximation methods," in $\mathrm{Nu}$ merical Techniques for Stochastic Systems, F. Archetti and M. Cugiani, Eds. Amsterdam, The Netherlands: North-Holland, 1980, pp. 395-406.

[12] H. J. Kushner and D. A. Clark, Stochastic Approximation Methods for Constrained and Unconstrained Systems. New York: Springer, 1978.

[13] M. B. Nevelson and R. Z. Hasminski, Stochastic Approximation and Recursive Estimation, vol. 47, Translations of Math. Monograph. AMS, 1996.

[14] J. C. Spall, "Multivariate stochastic approximation using a simultaneous perturbation gradient approximation," IEEE Trans. Automat. Contr., vol. 37, pp. 332-341, 1992.

[15] I. J. Wang, E. K. P. Chong, and S. R. Kulkarni, "Equivalent necessary and sufficient conditions on noise sequences for stochastic approximation algorithms," Advances Appl. Probability, vol. 23, pp. 784-801, 1996.

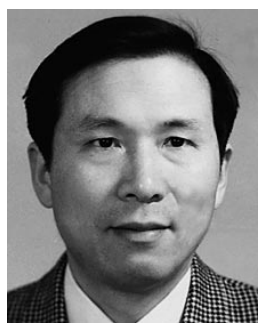

H. F. Chen (SM'94-F'97) received the Diploma from the Department of Mathematics and Mechanics, Leningrad (St. Petersburg) University, Russia.

He joined the Institute of Mathematics, Chinese Academy of Sciences, in 1961. Since 1979 he has been with the Institute of Systems Science, Chinese Academy of Sciences. He is a Professor of the Laboratory of System and Control and currently serves as the Director of the Institute. His research interests include stochastic systems, system identification, adaptive control, recursive estimation, and stochastic approximation. He has authored and coauthored more than 120 journal papers.

Mr. Chen is the Editor of Systems Science and Mathematical Sciences and is involved in the editorial boards of several international and domestic journals He was elected to the Chinese Academy of Sciences in 1993. He now serves as the President of the Chinese Association of Automation and is a Standing Member of the Council of the Chinese Mathematics Society. He also serves on the Technical Board of IFAC, chairing the Coordinating Committee Systems and Signals. For the 14th IFAC Congress to be held in 1999, he serves as the IPC Chairman and NOC Cochairman.

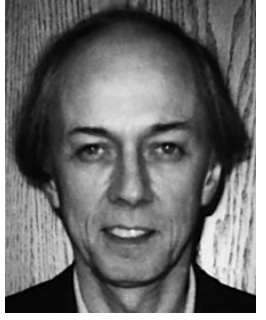

T. E. Duncan (M'92-SM'96-F'98) received the B.E.E. degree from Rensselaer Polytechnic Institute, Troy, NY, in 1963, and the M.S. and Ph.D. degrees from Stanford University, Stanford, CA, in 1964 and 1967, respectively.

He has held regular positions at the University of Michigan (1967-1971), the State University of New York, Stony Brook (1971-1974), and the University of Kansas, Lawrence (1974-present), where he is a Professor of Mathematics. He has held visiting positions at the University of California, Berkeley (1969-1970), the University of Bonn, Germany (1978-1979), and Harvard University, Cambridge, MA (1979-1980), and shorter visiting positions at numerous other institutions.

Dr. Duncan is an Associate Editor of SIAM Journal on Control and Optimization and is a member of AMS, MAA, and SIAM.

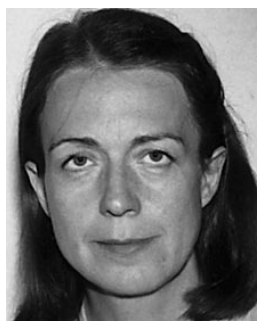

B. Pasik-Duncan (M'90-SM'95) received the M.S degree from the Mathematics Department of Warsaw University in 1970 and the Ph.D. and Habilitation degrees from the Mathematics Department of Warsaw School of Economics in 1978 and 1986, respectively.

She was a staff member of the Mathematics Department of the Warsaw School of Economics from 1970 to 1983 . In 1984 she joined the University of Kansas, where she is a Professor of Mathematics. Her research interests include adaptive control and its applications, stochastic theory and its applications, mathematics of finance, numberical methods in control, manufacturing systems, distributed parameter systems, mathematical statistics, and mathematical education.

Dr. Pasik-Duncan is currently serving as Program Director of the SIAM Activity Group on Control and Systems Theory and is a member of the SIAM Master Program Committee. She is an Associate Editor-at-Large of the IEEE TRANSACTIONS ON AUTOMATIC CONTROL and is currently serving as Chair of the Standing Committees on Assistance of Engineers at Risk and Women in Control. Her professional memberships include AMS, ASA, AWM, the Bernoulli Society, MAAA, the Polish Mathematical Society, SIAG/CST, and SIAM. 